\title{
Interesses Profissionais e Personalidade: análise correlacional a partir do ATPH e BFP
}

\author{
Professional Interests and Personality: correlational \\ analysis from BFP and ATPH
}

\author{
Ana Paula Porto Noronha \\ Universidade São Francisco, Brasil \\ Ce: ana.noronha@saofrancisco.edu.br
}

\author{
Camélia Santina Murgo Mansão \\ Universidade São Francisco,Brasil \\ Ce: camansao@hotmail.com
}

\author{
Maiana Farias Oliveira Nunes \\ Universidade São Francisco, Brasil \\ maiananunes@mac.com
}

Dirección postal: Rua Alexandre Rodrigues Barbosa, 45 - Centro, Itatiba, São Paulo/Brasil CEP 13253231

\begin{abstract}
Resumen. Este estudo objetivou explorar as relações entre interesses profissionais quando avaliado pelo modelo de Holland (RIASEC) e fatores de personalidade (Neuroticismo, Abertura, Realização, Extroversão e Socialização). Participaram 122 estudantes da primeira série do Ensino Médio, com idade entre 14 a 18 anos, sendo a maioria mulher (52\%). Aplicou-se os instrumentos ATPH - Avaliação dos Tipos Profissionais de Holland e BFP Bateria Fatorial de Personalidade. Foram encontradas associações significativas entre o fator Abertura e o tipo Artístico, bem como entre o fator Realização e os tipos Social e Empreendedor e o fator Socialização com o tipo Social. O fator Neuroticismo não mostrou associações significativas com as tipologias. Considerando-se os padrões de associação entre os construtos, discute-se a aplicabilidade para intervenções em Orientação Profissional.
\end{abstract}

Palabras clave: Avaliação psicológica, Orientação Profissional, Interesses profissionais, Personalidade, Adolescentes.

Abstract. This study aimed to explore the relationship between professional interests considering Holland's hexagonal model (RIASEC) and personality factors (Neuroticism, Openness, Conscientiousness, Extraversion and Agreeableness). 122 students took part on this study, who cursed first grade of high school, aging between 14 and 18 years old, mainly females (52\%). Two instruments were used: ATPH - Avaliação dos Tipos Profissionais de Holland and BFP - Bateria Fatorial de Personalidade. Significant correlations were found between the factor Openness and the Artistic type, as well as between the factor Conscientiousness and the Social and Enterprising types, and between the factor Agreeableness with the Social type. Neuroticism showed no significant correlation with the typologies. Considering the patterns of association found between the constructs, the results are discussed considering its aplicability for career interventions

Key Words: psychological assessment; career counseling; professional interests; personality; adolescents 


\section{Introdução}

Diversos têm sido os trabalhos encontrados na literatura nos quais estudiosos da Orientação Profissional buscam compreender os interesses profissionais e a estreita relação deles com as características de personalidade. Holland (1975) afirma que o interesse de uma pessoa para desenvolver determinadas atividades ocupacionais nada mais é do que a expressão de sua personalidade.

$\mathrm{Na}$ perspectiva teórica de Holland (1997), as preferências vocacionais vão sendo convertidas em interesses profissionais por meio da interação entre estímulos ambientais e aspectos motivacionais internos. Para o autor, seis tipos de interesses vocacionais podem ser descritos: Realista, Investigativo, Artístico, Social, Empreendedor e Convencional (conhecidos pela sigla RIASEC), de modo que existem características gerais associadas a cada tipo. Pessoas predominantemente realistas são pouco sociáveis e possuem baixa habilidade interpessoal, sendo assim, sentem-se pouco à vontade em situações sociais ou de conteúdo emocional, além de mostraremse conformistas, reservadas e poucos flexíveis. O tipo investigativo, embora mais aberto a questões subjetivas e abstratas, tende também a certa rigidez e pouco envolvimento emocional. As pessoas preferem atividades teóricas e autônomas, o que os torna ainda mais independente de vínculos grupais e mais centrados em si mesmos.

Já o tipo artístico é apreciador do contato interpessoal, podendo ter facilidade e desenvoltura ao se expressar. Adicionalmente, tende a ser empático e aberto a estímulos subjetivos e emocionais. As pessoas deste tipo valorizam os vínculos humanos e mostram sensibilidade para auxiliar; interesse para orientar, tratar e resolver as dificuldades dos outros. Gostam de se sentir aceitos e respeitados e de receber atenção em função de seu jeito expansivo. O tipo empreendedor, por sua vez, tem habilidades verbais e persuasivas; é extrovertido, demonstra iniciativa e tende a ser bem sucedido em posições de liderança. O tipo convencional tende a controlar os afetos e suas características principais são rigidez, conformismo, inibição, obediência, persistência e pouca criatividade. As descrições dos tipos indicam uma aproximação dos interesses com traços de personalidade, reforçando a ideia de que os interesses seriam a expressão da personalidade em uma área específica da vida, qual seja, do trabalho.

No que se refere especificamente ao construto personalidade, o modelo dos Cinco Grandes Fatores tem recebido atenção especial no âmbito estrangeiro (Costa \& Widiger, 2002; Digman, 1993, 2002; McCrae \& Costa, 1996). As características de personalidade, segundo este modelo, podem ser agrupadas dentro dos seguintes fatores: Extroversão, que diz respeito à quantidade de interações pessoais preferidas e aos estados de humor 
vivenciados de forma mais intensa; Socialização (ou Amabilidade) que se relaciona à orientação interpessoal ao longo de um contínuo da compaixão ao antagonismo; Realização (ou Conscienciosidade) expressa o grau de controle, organização e persistência; Neuroticismo referindo-se ao nível de ajustamento e instabilidade emocional; e Abertura à Experiência que diz respeito à tolerância e apreciação de novas experiências (Nunes, 2005).

Diversos estudos propuseram-se a verificar as relações entre os Cinco Grandes Fatores e os tipos de interesses vocacionais de Holland (Costa, McCrae, \& Holland, 1984; Gottfredson, Jones, \& Holland, 1993; Savickas, 1995; Swanson, 1995; Sullivan \& Hansen, 2004; Nauta, 2007; Valentine, Teodoro \& Balbinotti, 2009; Nunes \& Noronha, 2009a; Nunes \& Noronha, 2009b). Em seguida, encontram-se alguns achados.

No trabalho de Costa, McCrae e Holland (1984) foram avaliadas as associações entre os seis tipos de interesses vocacionais de Holland e os fatores Neuroticismo, Extroversão e Abertura à Experiência em uma amostra de 361 pessoas. Os resultados indicaram a existência de correlações fracas e moderadas, positivas e significativas, entre o fator Abertura e o tipo Investigativo e Artístico. Resultado semelhante foi observado entre o fator Extroversão e o tipo Social e Empreendedor. Apesar da hipótese dos pesquisadores de que o tipo Convencional deveria relacionar-se negativamente com o fator Abertura, não foram encontradas evidências para confirmação de tal suposição. O fator Neuroticismo não se correlacionou com nenhum dos tipos de Holland.

Um segundo estudo a ser apresentado é de autoria de Gottfredson, Jones e Holland (1993) no qual verificaram as correlações entre os seis tipos de interesses vocacionais de Holland e os Cinco Grandes Fatores, numa amostra de 725 recrutas da Marinha Americana. Os tipos Social e Empreendedor foram positivamente relacionados ao fator Extroversão. Ao lado disso, os tipos Artístico e Investigativo associaram-se significativamente ao fator Abertura.

Outros estudos foram desenvolvidos com vistas a investigar relações entre interesses e personalidade, como o de Nauta (2007) que investigou aspectos de carreira entre universitários. Foram participantes 115 sujeitos e os resultados apontaram correlações significativas entre interesses e personalidade. O tipo Realista relacionou-se positivamente com o fator Abertura e negativamente com o fator Neuroticismo. O tipo Investigativo não apresentou correlações significativas com os traços de personalidade. $\mathrm{O}$ tipo Artístico apresentou correlações significativas com Abertura, Socialização e Neuroticismo; o tipo Social com Socialização e Extroversão; o tipo Empreendedor com Extroversão e por fim, o tipo Convencional com Neuroticismo.

Estudos mais recentes como o de Valentine, Teodoro e Balbinotti (2009), realizado no Brasil, tem dado continuidade à investigação das 
relações entre interesses e personalidade considerando a tipologia de Holland e o modelo dos Cinco Fatores. Para tanto, os autores contaram com uma amostra de 145 estudantes, graduandos das áreas de Ciências Exatas (Engenharia Elétrica e Mecânica; Física), Ciências Contábeis, Biológicas e Saúde (Biologia, Educação Física, Enfermagem e Nutrição), e Humanas (Psicologia e Serviço Social). Os instrumentos utilizados foram o Inventário Tipológico de Interesses Profissionais- ITIP- 96 (Balbinotti, 2003), a Escala Fatorial de Neuroticismo (EFN) (Hutz \& Nunes, 2001), Escala Fatorial de Extroversão (EFEx) (Nunes \& Hutz, 2007a, 2007b) e Escala Fatorial de Abertura à Experiência (EFA) (Vasconcellos \& Hutz, 2008).

No estudo, em relação aos sexos, foram encontradas associações significativas, de magnitude de fraca a moderada entre o fator Abertura e o tipo Investigativo, ou seja, pessoas com características de personalidade abertas e tolerantes a novas experiências, pró-ativas, curiosas e imaginativas tendem a manifestar preferências por atividades investigativas (próprias de profissões como pesquisador, médico e dentista) e artísticas (pintor artístico, cantor e escritor).

Apenas para a amostra feminina, os fatores de Depressão (Neuroticismo) e Comunicação (Extroversão) associaram-se negativamente, ainda que de maneira fraca, aos tipos Empreendedor e Convencional. Isso torna possível afirmar que quanto maior a presença de características como desesperança e depressão em mulheres, menor seria o interesse por atividades empreendedoras. O subfator Altivez (Extroversão) associou-se de forma positiva e moderada com os tipos Artístico e Empreendedor, apenas para participantes do sexo masculino, permitindo inferir que aqueles que se percebem como grandiosos e pouco modestos manifestam interesses por atividades de gerenciamento e chefia (Valentine, Teodoro \& Balbinotti, 2009).

Com vistas a compreender as relações entre interesses e personalidade, Nunes e Noronha (2009) contaram em seu estudo com uma amostra de 115 adolescentes, estudantes de uma escola do estado do Paraná. As autoras utilizaram como instrumentos o Self-Directed Search (Holland, Johnston \& Asama, 1994) e a Bateria Fatorial de Personalidade (Nunes, Hutz \& Nunes, 2010). Foram encontradas associações positivas, sendo as mais elevadas entre o fator Abertura e os tipos Artístico e Social. O fator Extroversão correlacionou-se positivamente com o tipo Empreendedor, revelando pessoas que gostam de estar em grupos, de falar em público e de exercer atividades diárias que tenham dinamismo. Ainda, foram encontrados coeficientes positivos ente o fator Socialização e o Tipo Social, o que pode representar aspectos de generosidade, altruísmo e confiança nas pessoas. $\mathrm{O}$ único fator que não apresentou correlação significativa foi Neuroticismo. Resultados semelhantes foram encontrado no estudo de Nunes e Noronha (2009b), ao pesquisarem os interesses profissionais e traços de personalidade 
em 211 adolescentes brasileiros, com os mesmos instrumentos recémmencionados (SDS e BFP). Ao utilizar uma análise fatorial de segunda ordem, as autoras observaram aproximações entre os tipos Artístico e os fatores Abertura e Extroversão; o tipo Social com Socialização; enquanto os fatores Neuroticismo e Realização não se associaram de forma acentuada aos interesses profissionais.

Considerando que os interesses profissionais possuem estreita relação com as escolhas profissionais realizadas, mostra-se pertinente o presente estudo, assim como a análise da relação com traços de personalidade. Desse modo, esta pesquisa teve como objetivo investigar a associação entre fatores de personalidade e interesses profissionais.

\section{Participantes}

\section{Método}

Colaboraram para o desenvolvimento do estudo 122 estudantes brasileiros da primeira série do Ensino Médio de duas escolas, sendo uma pública e outra particular, localizadas no interior do Estado de São Paulo. As idades dos participantes variaram de 14 a 18 anos $(D P=1,34 ; M=15,6)$. No que se refere ao sexo, $57(48 \%)$ eram homens e $65(52 \%)$, mulheres. A escolha da amostra foi feita por conveniência, em função de contatos prévios com a escola e a disponibilidade demonstrada para a realização da pesquisa. Considerando os objetivos do estudo, de verificar as associações entre interesses profissionais e personalidade, considerou-se o tamanho da amostra adequado para as análises pretendidas, tal como pontuado por Levin e Fox (2004).

\section{Instrumentos}

Para avaliação dos interesses profissionais, foi usado o ATPHAvaliação dos Tipos Profissionais de Holland (Primi, Muniz, Nunes \& Mansão, 2008), elaborado a partir do SDS - Self Directed Search - de Holland (1975). Esse instrumento tem como objetivo verificar interesses e preferências profissionais, com vistas à elaboração de um perfil tipológico. É composto por 154 itens que representam as seis tipologias de Holland: Realista, Investigativo, Artístico, Social, Convencional e Empreendedor. As respostas mostram a intensidade do interesse do sujeito pelas atividades profissionais descritas nos itens. Cada item é avaliado por uma escala de quatro pontos, do tipo Likert, correspondendo a "nenhum interesse" (resposta 1) a "muito interesse" (resposta 4). As evidências de validade do ATPH foram investigadas por Mansão e Noronha (no prelo) a partir de uma amostra de 462 estudantes de Ensino Médio, com idade média de 16 anos $(D P=1,34) . \quad \mathrm{Na}$ análise da precisão, avaliada pelo índice de consistência 
interna de cada uma das tipologias do modelo RIASEC, calculando-se o Alfa de Cronbach $(\alpha)$, cada uma das tipologias apresentou índices muito satisfatórios: Realista $(0,95)$, Investigativo $(0,93)$, Artístico $(0,94)$, Social $(0,96)$, Empreendedor $(0,95)$ e Convencional $(0,96)$. A Análise Fatorial, realizada por meio do método de componentes principais, mostrou seis fatores correspondendo à idéia original das tipologias propostas por Holland (1975). Para tanto, foram considerados os índices de saturação superiores a 0,40 .

O tipo Realista, característico de sujeitos com habilidades mecânicas e características de praticidade e conservadorismo, foi representado no fator 1. Já as características reveladoras de sujeitos analíticos e curiosos (tipo Investigativo), pelo fator 2. O tipo Artístico (predomínio da criatividade e do uso da imaginação) foi identificado no fator 3. A tipologia Social, comum entre indivíduos que se realizam ajudando outras pessoas e preocupando-se com o bem estar humano, foi identificada no fator 4. O Fator 5 representou a tipologia Empreendedora (preferência por exercer papéis de poder e de liderança) e o fator 6, a tipologia Convencional (pessoas organizadas e metódicas).

O outro instrumento utilizado foi a Bateria Fatorial de Personalidade BFP (Nunes, Hutz \& Nunes, 2010), composto por 126 itens que descrevem sentimentos, opiniões e atitudes. Os itens contemplam Neuroticismo, Extroversão, Socialização, Realização e Abertura para experiência. As respostas são registradas numa escala likert de 7 pontos (de 1 a 7), de acordo com o quanto o sujeito se identifica com cada sentença. $O$ instrumento objetiva avaliar a personalidade e foi construído com base no modelo dos cinco fatores (CGF). Para o estudo normativo, contou-se com uma amostra de 6.599 pessoas, sendo a maioria estudantes universitários ou de ensino médio, provenientes de 11 estados brasileiros. As análises fatoriais exploratórias indicaram a presença de cinco fatores, em concordância com o modelo adotado. A precisão dos fatores variou entre 0,74 (Abertura a experiências) e 0,89 (Neuroticismo), considerando o alfa de Cronbach. O manual do teste apresenta estudos de precisão para grupos específicos considerando sexo, nível educacional e tipo de estudo (grupos específicos pesquisados, como idosos ou pacientes internados em hospitais). Adicionalmente, há a descrição de diversos estudos de validade, tendo esse instrumento parecer favorável do Conselho Federal de Psicologia.

$\mathrm{O}$ fator Extroversão refere-se à quantidade e à intensidade das interações interpessoais preferidas, nível de atividade, necessidade de estimulação e capacidade de alegrar-se. Socialização, embora também represente uma dimensão interpessoal, avalia o interesse pelo bem-estar dos outros, confiança nas pessoas e adesão a regras ou normas sociais. Já o fator Realização faz referência ao grau de organização, persistência, controle e motivação para alcançar objetivos enquanto que o fator Neuroticismo diz 
respeito ao nível de ajustamento emocional e instabilidade, envolvendo especificamente a vulnerabilidade à opinião dos outros, instabilidade de humor/emocional, presença de sintomas depressivos e comportamentos passivos ou falta de energia para agir em situações importantes. O último fator, denominado Abertura para experiências, reflete comportamentos exploratórios e de reconhecimento da importância de vivenciar novas experiências, avaliando interesse por novas idéias e busca por novidades (Nunes, Hutz \& Nunes, 2010).

\section{Procedimentos}

Anterior à realização da pesquisa, o projeto de pesquisa foi encaminhado para um Comitê de Ética em Pesquisa. Após aprovação, iniciou-se o procedimento de coleta de dados, que teve a duração de quatro meses, no período de novembro de 2009 a fevereiro de 2010. Após contato com as escolas e posterior autorização da direção, houve um contato também com os estudantes, com a intenção de informar os objetivos da pesquisa e entregar o Termo de Consentimento Livre e Esclarecido (TCLE) para que fossem encaminhados aos responsáveis. Posteriormente, os TCLEs assinados foram recolhidos e a aplicação dos instrumentos deu-se de maneira coletiva, em dia e horário previamente estabelecidos. Para a análise de dados, usou-se a correlação de Pearson entre o ATPH e a Bateria Fatorial de Personalidade (BFP). Para as análises, utilizou-se o programa estatístico SPSS (versão 16.0 for Windows). Primeiramente será exposta a correlação dos tipos de interesses com os cinco fatores e, na sequência, serão exploradas as correlações dos mesmos com as facetas de personalidade.

\section{Resultados e Discussão}

Buscou-se verificar associações entre as tipologias propostas pelo ATPH e os fatores de personalidade da bateria. Os resultados são descritos na Tabela 1.

De modo geral as correlações significativas variaram entre muito baixas $(r=-0,19)$ a moderadas $(r=0,40)$. Assim, reforça-se a hipótese de que se trata de construtos distintos que, em alguns casos, possuem aproximações, sendo a personalidade um fenômeno mais amplo para descrever as pessoas, em comparação com os interesses profissionais. Dentre os coeficientes significativos, o tipo Social teve mais associações significativas com os fatores de personalidade, especificamente com Socialização $(r=0,33)$, Extroversão e Realização ( $r=0,21$, para ambos), embora em todos os casos com magnitudes baixas. Nunes e Noronha (2009a) encontraram resultados semelhantes em indivíduos que revelaram, ao mesmo tempo, desejo pelo bem-estar aos outros, e prazer em realizar ações de cunho assistencial (tipo 
Social) e desejo de trabalhar com atividades de cuidado na área da saúde (dimensão Ciências Biológicas e da Saúde). Adicionalmente, a preocupação com os outros e o gosto pelo cumprimento de regras sociais, características dos traços de personalidade do fator Socialização, também foram evidenciados nos participantes do estudo.

Tabela 1.

Correlações entre ATPH e fatores da BFP

\begin{tabular}{cccccc}
\hline \multirow{2}{*}{ ATPH } & \multicolumn{5}{c}{ BFP } \\
\cline { 2 - 6 } & Neuroticismo & Extroversão & Socialização & Realização & Abertura \\
\hline Realista (R) & 0,03 & $-0,00$ & $-0,19^{*}$ & 0,00 & $-0,11^{*}$ \\
Investigativo (I) & 0,04 & $-0,06$ & $-0,02$ & 0,15 & $-0,03$ \\
Artístico (A) & 0,07 & 0,12 & 0,02 & $-0,01$ & $0,40^{* *}$ \\
Social (S) & $-0,08$ & $0,21^{*}$ & $0,33^{* *}$ & $0,21^{*}$ & 0,12 \\
Empreendedor(E) & $-0,15$ & 0,17 & $-0,06$ & $0,26^{* *}$ & $0,20^{*}$ \\
Convencional (C) & $-0,13$ & 0,12 & $-0,07$ & 0,15 & $-0,08$ \\
\hline
\end{tabular}

Nota. ${ }^{*} p<0,05 ;{ }^{* *} p<0,01$

Já o tipo Empreendedor apresentou correlações significativas com o escore geral de Realização $(r=0,26)$ e Abertura $(r=0,20)$. Assim, pessoas que apresentam tendência a envolver-se com atividades profissionais que prescindem de persuasão, argumentação e uso da influência social para atingir objetivos do trabalho, em geral são responsáveis, possuem clareza de objetivos, e também mostram-se mais abertas para novas ideias e experiências. É possível que a abertura a ideias, ações e valores sociais facilite a interação de pessoas com alto escore no tipo Empreendedor com outras sem estas características, o que pode facilitar o trabalho.

Por sua vez, o tipo Realista apresentou correlações significativas e negativas com os fatores Socialização e Abertura (em ambos os casos, com $r$ $=-0,19)$. Pode-se inferir que pessoas que gostam de trabalhar com problemas concretos, que exijam pouca interação social apresentam uma tendência a não se preocupar com o bem-estar dos outros (baixa Socialização) e a não buscar experiências ou ideias novas (baixa Abertura). O estudo de Nunes e cols (2010) também encontrou correlação significativa e negativa $(r=-0,15)$ entre o tipo Realista e o fator Socialização, ainda que muito baixa, porém o mesmo não ocorreu com o fator Abertura, que não apresentou correlação significativa com esse tipo de interesse.

Dentre os tipos avaliados pelo ATPH, o Artístico foi o que apresentou a correlação mais alta com os fatores de personalidade, especificamente com o escore total de Abertura $(r=0,40)$. No estudo de Black e Sackette (1999) também em relação a esse resultado foram encontradas correlações entre 0,27 e 0,39 entre o tipo Artístico e o fator Abertura. 
As tipologias Convencional e Investigativo não apresentaram correlações significativas com os fatores da BFP. Esse resultado é parcialmente semelhante ao de Nunes e cols (2010), que também não verificaram correlações significativas entre o tipo Convencional e os cinco fatores de personalidade, porém o tipo Investigativo correlacionou-se significativamente com o fator Realização $(r=0,20)$. Entre os fatores de personalidade, Neuroticismo não apresentou correlação significativa com nenhum tipo de interesse, o que também foi encontrado em outros estudos (Costa, McCrae, \& Holland, 1984; Gottfredson, Jones, \& Holland, 1993; Nunes \& Noronha, 2009a; Nunes \& Noronha, 2009b). Assim, é possível que o fator Neuroticismo seja mais útil para investigar outras questões do desenvolvimento vocacional, tal como a indecisão profissional (Amir, Gati, \& Klieman, 2008; Saka, Gati \& Kelly, 2008; Nunes, Noronha, Nunes \& Primi, 2010).

De modo geral, foram encontradas aproximações com os resultados da investigação de Nauta (2007), com 115 universitários, avaliados com instrumentos baseados no modelo RIASEC e na teoria dos Cinco Grandes Fatores de Personalidade. Especificamente, o tipo Convencional relacionouse apenas com Neuroticismo $(r=0,32)$. Os demais resultados de Nauta (2007) estão em consonância com os resultados desse estudo, ou seja, o tipo Social correlacionou-se com Socialização $(r=0,40)$ e Extroversão $(r=0,42)$ e por fim o tipo Artístico com Abertura $(r=0,44)$.

No presente estudo procedeu-se a uma segunda análise para a qual foram consideradas as facetas de cada fator da BFP e as tipologias do ATPH. A Tabela 2 possibilita a visualização dos resultados.

Foi possível verificar que as facetas de Extroversão se correlacionaram com os interesses Social, Empreendedor, Artístico e Convencional, em todos os casos com coeficientes positivos. Os achados permitem inferir que nessa dimensão tendem a se destacar interesses por tarefas ocupacionais que envolvam a expressão de idéias e interação com pessoas, já que uma faceta que se destacou foi Comunicação $(r=0,28 \mathrm{com}$ o tipo Social; $r=0,21 \mathrm{com}$ Empreendedor e $r=0,19$ com Artístico). Resultado coerente também se observou em relação às atividades que utilizam do convencimento e liderança de pessoas, na faceta Dinamismo $(r=0,28$ com tipo Empreendedor e $r=0,21$ com o Convencional). 
Tabela 2.

Correlações entre ATPH e facetas de cada fator da BFP

\begin{tabular}{|c|c|c|c|c|c|c|}
\hline \multirow[b]{2}{*}{ BFP } & \multicolumn{6}{|c|}{ ATPH } \\
\hline & $\mathrm{R}$ & I & $\mathrm{A}$ & $\mathrm{S}$ & $\mathrm{E}$ & $\mathrm{C}$ \\
\hline N1 - Vulnerabilidade & 0,01 & 0,02 & $-0,04$ & $-0,16$ & $-0,15$ & $-0,15$ \\
\hline N2 - Instabilidade & $-0,08$ & 0,09 & $-0,00$ & 0,09 & $-0,16$ & $-0,08$ \\
\hline N3- Falta de Energia & 0,10 & $-0,01$ & $0,22^{*}$ & $-0,07$ & $-0,05$ & $-0,12$ \\
\hline N4 - Depressão & 0,07 & 0,03 & 0,01 & $-0,13$ & $-0,08$ & $-0,05$ \\
\hline E1 - Comunicação & 0,00 & $-0,04$ & $0,19^{*}$ & $0,28^{* *}$ & $0,21^{*}$ & 0,11 \\
\hline E2 - Altivez & $-0,07$ & $-0,01$ & 0,04 & 0,01 & 0,12 & 0,11 \\
\hline E3 - Dinamismo & 0,01 & 0,00 & 0,08 & 0,15 & $0,28^{* *}$ & $0,20^{*}$ \\
\hline E4 - Interação social & $-0,13$ & $-0,15$ & 0,04 & 0,17 & $-0,11$ & $-0,07$ \\
\hline S1 - Amabilidade & $-0,17^{*}$ & $-0,00$ & 0,12 & $0,36^{* *}$ & $-0,01$ & $-0,15$ \\
\hline S2 - Pró-Sociabilidade & $-0,17$ & 0,08 & $-0,05$ & $0,26^{* *}$ & 0,01 & 0,09 \\
\hline S3 - Confiança & $-0,08$ & $-0,11$ & $-0,02$ & 0,10 & $-0,13$ & $-0,11$ \\
\hline R1 - Competência & $-0,06$ & 0,13 & $-0,03$ & $0,25^{* *}$ & $0,27^{* *}$ & $0,29^{* *}$ \\
\hline R2 - Ponderação & 0,03 & $-0,02$ & $-0,05$ & 0,10 & 0,06 & $-0,06$ \\
\hline R3- Empenho & 0,03 & $0,25^{* *}$ & $-0,03$ & 0,15 & $025^{* *}$ & 0,14 \\
\hline A1 - Interesse por novas idéias & $-0,10$ & 0,10 & $0,49^{* *}$ & 0,13 & $0,21^{*}$ & $-0,11$ \\
\hline A2 - Liberalismo & $-0,10$ & 0,04 & $0,19^{*}$ & 0,17 & $0,21^{*}$ & 0,10 \\
\hline A3 - Busca por novidades & $-0,15$ & $-0,21^{*}$ & 0,08 & $-0,03$ & $-0,02$ & $-0,15$ \\
\hline
\end{tabular}

Nota. ${ }^{*} p<0,05 ;{ }^{* *} p<0,01$ 
Em relação à Socialização, foram encontradas associações positivas entre Amabilidade e Pró-sociabilidade e o tipo Social, o que indica que quanto maior o interesse por profissões caracterizadas pela ajuda e cuidado aos outros, maior possibilidade de escores elevados em itens que descrevem comportamentos de gentileza, preocupação com os demais, cumprimento das regras sociais, confiança na boa intenção das pessoas, entre outros (Nunes, \& cols, 2010). Vale ressaltar que a faceta Amabilidade obteve correlação negativa com o tipo Realista, resultado que também corrobora o estudo de Nunes e cols (2010). Para os autores, isso se justifica pelo fato de que pessoas com interesses por ocupações que exigem o uso da criatividade, da imaginação e de pouco envolvimento com tarefas mecânicas tendem a buscar atividades ocupacionais direcionadas para o cuidado com o outro, justamente pela sua característica de amabilidade.

Por sua vez, as facetas do fator Realização apresentaram correlações significativas, embora baixas, especificamente entre a faceta Competência e os tipos Social, Empreendedor e Convencional e da faceta Empenho com o tipo Investigativo e Empreendedor. Assim, verifica-se que a característica de se sentir competente e capaz de atingir seus objetivos (faceta Competência) está presente em tipos adjacentes, enquanto o comprometimento com as tarefas e empenho para atingir as metas mostra-se mais destacado apenas em profissões de cunho mais científico (tipo Investigativo) ou mais ligadas aos negócios e à busca por atingir resultados tangíveis por meio do trabalho (tipo Empreendedor).

Quanto às facetas de Abertura, pode-se verificar que tanto abertura a novas ideias como Liberalismo associaram-se significativamente com os tipos Artístico e Empreendedor. Em outros estudos que também foram observadas correlações entre Abertura e tipo Artístico (Holland, Johnston, \& Asama, 1994; Sullivan \& Hansen, 2004; Nunes \& Noronha, 2009a; Nunes \& Noronha, 2009b; Valentine, Teodoro \& Balbinotti, 2009; Nunes, Hutz \& Nunes, 2010), indicando que pessoas com grande abertura a novas idéias, criativas, que gostam de novidades e são mais liberais tendem a se interessar pelas profissões representadas pelo tipo Artístico. Houve uma correlação negativa entre a faceta Busca por novidades e o tipo Investigativo, sugerindo que quanto mais comportamentos exploratórios de busca por contato com situações novas, menor o interesse por profissões do tipo Investigativo, o que pode ser interpretado no sentido de que o tipo Investigativo se interessa muito mais por novidades no campo das ideias do que das ações, mostrando-se mais apegado à rotina.

A análise de Neuroticismo apontou apenas uma correlação significativa entre a faceta Falta de energia e o Tipo Artístico. De modo geral, o fator igualmente não se mostrou relevante em outras pesquisas (Costa, McCrae \& Holland 1984; Gottfredson, Jones \& Holland, 1993; Tokar \& Swanson, 1995; Schinka, Dye \& Curtiss, 1997; Armstrong \& Anthoney, 2009). 
Ainda, cabe citar Armstrong e Anthoney (2009) que conduziram um estudo para avaliar as relações entre personalidade e interesses, no qual chamam a atenção para a importância de investigar os subfatores da personalidade para aprofundar a compreensão do tema. De maneira geral, constataram que as relações entre todos os subfatores do fator Extroversão e os tipos empreendedor e Social puderam ser apontadas como robustas, bem como entre o fator Abertura e o tipo Artístico. Na mesma direção, o fator Socialização/Amabilidade correlacionou-se com o tipo Social. Já para o fator Neuroticismo os resultados indicaram inexpressividade na associação com os tipos de Holland. Tais achados assemelham-se aos do presente estudo.

\section{Considerações Finais}

As descobertas trazidas a partir do estudo reforçam outros achados de pesquisas que revelaram a relevância dos construtos interesses e personalidade nos processos de Orientação Profissional. A comparação dos tipos profissionais de Holland com os fatores do modelo dos Cinco Grandes Fatores de Personalidade apontou resultados que sugerem relações entre os construtos. Em linhas gerais, as associações significativas mais fortes foram encontradas entre o fator Abertura e o tipo Artístico, bem como o fator Socialização com o tipo Social. O fator Neuroticismo e os tipos de interesses não mostraram associações relevantes, conforme esperado.

Espera-se que a análise combinada de interesses e personalidades possa trazer informações para os participantes de processos de OP, clarificando suas escolhas e favorecendo o delineamento de um projeto profissional. Conhecer os padrões de interação entre interesses profissionais e traços de personalidade mostra-se relevante no sentido de ampliar as possibilidades de intervenções de carreira que estimulem o autoconhecimento (exploração das características pessoais) e que facilitem a tomada de decisão de carreira. Desse modo, reforça-se a ideia de que a gratificação da estrutura de inclinações motivacionais, em atividades laborais ou cotidianas, seria um elemento favorecedor da saúde mental, representando um aspecto de preservação da própria vida.

Em síntese, trabalhos científicos dessa natureza merecem ser desenvolvidos com maior freqüência, já que a compreensão das relações entre os elementos investigados podem levar ao aprimoramento dos processos de OP e favorecer a integração entre teoria e prática na área, necessidade essa que já vem sendo apontada por importantes estudiosos como Savickas e Walsh (1996). Ainda no tocante ao presente estudo, é fundamental atentar para a necessária prudência na generalização dos dados encontrados, uma vez que trabalhou uma amostra restrita e que se trata de um dos primeiros estudos com o instrumento de interesses utilizado. 


\section{Referencias}

Amir, T., Gati, I., \& Klieman, T. (2008). Understanding and interpreting Career Decision-Making Difficulties. Journal of Career Assessment, 16(3), 281-309.

Armstrong, P. I., \& Anthoney, S. F. (2009). Personality facets and riasec interests: An integrated model. Journal of Vocational Behavior, 75(9), 346-359.

Balbinotti, M. A. A. (2003). Inventário Tipológico de Interesses Profissionais. São Leopoldo: Núcleo de Orientação Vocacional, Universidade do Vale do Rio dos Sinos

Black, R.J. \& Sackette, (1999). Holland's Typollogy and the Five-Factor Model: A Rational-Empirical Analisys. Journal of Career Assesement, 7 (3), 249-279.

Costa, P. T., McCrae, R. R., \& Holland, J. L. (1984). Personality and vocational interests in an adult sample. Journal of Applied Psychology, 69(3), 390-400.

Costa, P. T. Jr. \& Widiger, T. A. (2002). Introduction. Em: P. T. Costa \& T. A. (Eds.). Personality Disorders and the Five-Factor Model of Personality. (2., cd. pp . 316). Washington, D.C: American Psychology Association.

Digman, J. M. (1993). Historical antecedents of the five-factor model. In P. T. Costa \& T. A. Widger (Eds.), Personality disorders and the five-factor Model of Personality (pp. 13-18). Washington, DC: American Psychological Association.

Digman, J. M. (2002). Historical antecedents of the five-factor model. In P. T. Costa \& T. A. Widger (Eds.), Personality disorders and the five-factor Model of Personality (2a ed., pp. 17-22). Washington, DC: American Psychological Association.

Gottfredson, G. D., Jones, E. M., \& Holland, J. L. (1993). Personality and vocational interests: The relation of Holland's interest dimensions to five robust dimensions of personality. Journal of Couseling Psychology, 40(4), 518-524.

Holland, J. L. . (1975). Técnica de la elección vocacional: tipos de personalidd y modelo ambientales. (R. D. Guerrero, Trad.). México: Trillas.

Holland, J. L., Johnston, J. A., \& Asama, N. F. (1994). More Evidence for the Relationship

Between Holland's Personality Types and Personality Variables. Journal of Career Assessment, 2(4), 331- 340.

Holland, J. L. (1997). Making vocational choices: A theory of vocational personalities and work environments ( $3 \mathrm{a}$ ed.). Odessa, FL: Psychological Assessment Resources.

Hutz, C. S., \& Nunes, C. H. (2001). Escala Fatorial de Ajustamento Emocional/ Neuroticismo (EFN). São Paulo: Casa do Psicólogo.

Levin, J., \& Fox, J. A. (2004). Estatística para Ciências Humanas. São Paulo: Prentice Hall (Pearson)

McCrae, R. R., \& Costa, P. T., Jr. (1996). Toward a new generation of personality theories: Theoretical contexts for the five-factor model. In J. S. Wiggins (Ed.), The five-factor model of personality: Theoretical perspectives (pp. 51-87). New York: Guilford.

Nauta, M. M. (2007). Career Interests, Self-Efficacy and Personality as Antecedents off Career Exploration. Journal of Career Assessment , 15 (2), 162-180.

Nunes, C. H. S. S. (2005). Construção, normatização e validação das escalas de socialização e extroversão no Modelo dos Cinco Grandes Fatores. Tese de Doutorado nãopublicada, Universidade Federal do Rio Grande do Sul, Porto Alegre. 
Nunes, C. H. S. S. \& Hutz, C. S. (2007a). Escala Fatorial de Extroversão: Manual Técnico. São Paulo: Casa do Psicólogo.

Nunes, C. H. S. S. \& Hutz, C. S. (2007b). Escala Fatorial de Socialização: Manual Técnico. São Paulo: Casa do Psicólogo.

Nunes, C.H.S. ; Hutz, C. S.; Nunes, M. O. (2010). Bateria Fatorial de Personalidade Manual Técnico. Casa do Psicólogo: São Paulo.

Nunes, M. F. O., \& Noronha, A. P. P. (2009a). Interesses e personalidade: um estudo com adolescentes em orientação profissional. Revista Galego-Portuguesa de Psicoloxía e Educación, 17(1,2), 115-129.

Nunes, M. F. O., \& Noronha, A. P. P. (2009b). Relações entre interesses, personalidade e habilidades cognitivas: um estudo com adolescentes. PsicoUSF, 14(1), 131-141.

Nunes, M. F. O., Noronha, A. P. P., Nunes, C. H. S. S., \& Primi, R. (2010). Dificuldades para decisão profissional e traços de personalidade em adolescentes. In C. Hutz (Ed.), Avanços em Avaliação Psicológica e Neuropsicológica de crianças e adolescentes (pp. 309-336). São Paulo: Casa do Psicólogo.

Primi,R.; Muniz, M.; Nunes, M.; e Mansão, C.M. (2008). Avaliação dos Tipos Profissionais de Holland - ATPH. Relatório Técnico não publicado

Saka, N., Gati, I., \& Kelly, K. R. (2008). Emotional and personality-related aspects of career decision making difficulties. Journal of Career Assessment, 16(4), 403-424. doi:10.1177/1069072708318900

Savickas, M. L. (1995). Examining the personal meaning of inventoried interests during career Counseling. Journal of Career Assessment, 3(2), 188-201.

Savickas, M.L.; Walsh, W.B. (1996). Handbook of career counseling theory and practice (pp. 191-208). Palo Alto, CA: Davies-Black.

Schinka, J. A., Dye, D. A., \& Curtiss, G. (1997). Correspondence between five-factor and RIASEC models of personality. Journal of Personality Assessment, 68(2), 355368.

Sullivan, B. A., \& Hansen, J. C. (2004). Mapping Associations between interests and personality: Toward a conceptual understanding of individual differences in vocational behavior. Journal of Counseling Psychology, 51(3), 287-298.

Swanson, J. (1995). The Process and Outcomes oh Career Counseling. In S.H.Osipow \& W. B. Walsh (Eds.). Handbook of Vocational Psychology, Theory Research, and practice. (2th ed., pp. 217-260). Mahwah, NJ: Lawrence Erlbaum.

Tokar, D. M., \& Swanson, J. L. (1995). Evaluation of the correspondence between Holland's Vocational Typology and the Five-Factor Model of Personality. Journal of Vocational Behavior, 46(1), 89-108.

Valentini, F.; Teodoro, M. L. M.; Balbinotti, M. A. A. (2009). Relações entre interesses vocacionais e fatores de personalidade. Revista Brasileira de Orientação Profissional, 10(2), 57-68.

Vasconcellos, S. J. L., \& Hutz, C. S. (2008). Construção e validação de uma escala de abertura à experiência. Avaliação Psicológica, 7, 135-141.

Recibido: 16 de octubre de 2011

Aceptado: 24 de febrero de 2012 